\section{Consciousness and Computers}

Neville Holmes, University of Tasmania

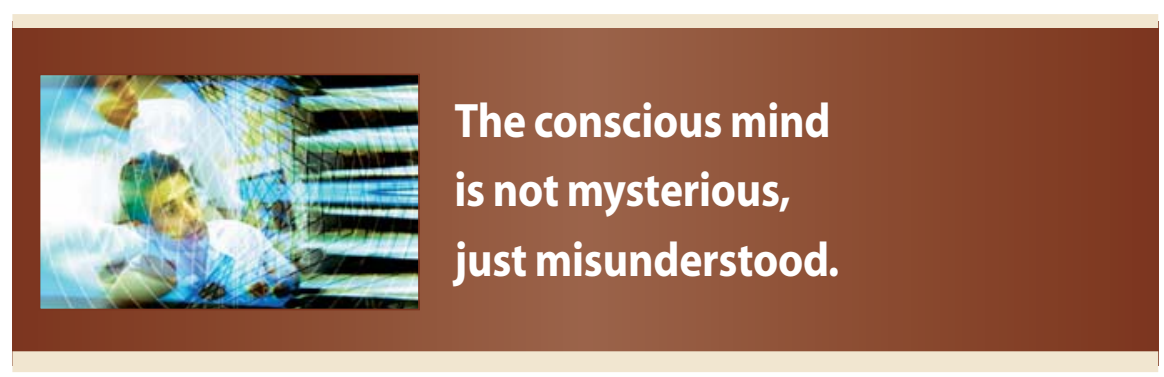

R ecently, the cover of an issue of Time (29 Jan. 2007) that appeared to promote ancient phrenology caught my attention. Closer inspection showed it to be a "Mind \& Body Special Issue." This surprised me because The Economist's special Christmas/New Year issue (23 Dec. 2006) had featured the supplement "A Survey of the Brain." So I bought the copy of Time to compare with the earlier issue of The Economist.

Although both started with a signed introduction, the rest of the content was stylistically opposed. The Economist had five anonymous reports compiled from interviews with, and quotations of, experts, the lot decorated with a few drawings and diagrams. Time had 10 richly illustrated essays informed and often written by experts, and followed by a short puzzle section. The contest, if indeed it was one, seemed to be a standoff, like a saber versus a shillelagh.

The computing profession is relevant here. For example, certain aspects of computer architecture can be used to explain the functioning of the brain and its mind, and I tried to do this in an earlier column ("Would a Digital Brain Have a Mind?" Computer, May 2002, pp. 112, 110-111), inspired by an encounter with philosophers contemplating the nature of the mind.

One issue that seems to have won general acceptance holds that the mind is a process hosted by the substance of the brain and its nervous system. The mind is like the execution of a program, the brain the machine that holds the program and carries out the processing it specifies.

The most prominent topic in both publications was consciousness. Time's lead article referred to "a bracing new field, the science of consciousness." The Economist's lengthy article on the topic referred to "what is, perhaps, the greatest mystery of the human brain: the nature of consciousness." Judging by the content of these two magazines, however, they appear to be promoting a neodualism, one that seems to contrast the conscious and unconscious mind and to dismiss lower animals as unconscious. The nature of computers can help illuminate these issues.

\section{CONSCIOUSNESS}

Time's lead article focused on two problems this new science faces-one easy and one hard-as dubbed by philosopher David Chalmers. The Easy Problem (their capitals) "is to distinguish consciousness from unconscious mental computation, identify its correlates in the brain and explain why it evolved. ... The Hard Problem is explaining how subjective experience arises from neural computation."

Behind both problems lies the assumption that two kinds of mentality exist, with the brain divided into two parts, one hosting conscious thought and the other unconscious, presumably much as old mainframe architectures shared processing between a central processing unit and peripheral device control units.

This analogy holds some relevance. Just as a disk control unit will, with a little negotiation with the CPU, manipulate data flowing between disks and the main store, so do the retinas in the eye work on the data provided by its receptors to analyze and greatly compress that data before sending it along the optic nerve.

Awareness or alertness is one kind of consciousness, and it has two aspects. We judge other people to be conscious by how they behave, that is, by their motor behavior. Until recently, we judged people in a vegetative state to be unconscious. Now, magnetic resonance imaging has shown that the brains of at least some of these unfortunates react to stimulus just as an ordinary person's brain does. They are like a mainframe computer with a broken output controller.

We judge ourselves to be conscious by our awareness of sensations. However, this consciousness is not an on-or-off phenomenon because we have degrees of awareness. We can be wide awake or drowsy or somewhere in between. We drift into sleep, then become unconscious to different degrees at different stages, characterized by ease of arousal. Sleep is unlike the unconsciousness brought about by a concussion, from which there is no immediate arousal. This unconsciousness is like the state of a computer with no battery and its power supply turned off, while the unconsciousness of sleep is like the Continued on page 98 
sleep state of the laptop I'm writing this essay on, which has two levels.

Another aspect of consciousness is its capacity for awareness rather than its awareness at any time. There are those who would like to see this aspect as binary to distinguish between clever animals like humans who are aware of themselves and "lower" beings that are not. The popular test for this is to put a mirror in front of the subjects to be tested and observe if they are aware of looking at themselves. Clearly, this is a kind of intelligence test, and it was widely thought that only humans and maybe chimpanzees could pass it. Lately, the number of species that pass the test has been growing and, in any case, the observer imposes the binarity.

\section{THE EASY PROBLEM}

The first part of The Easy Problem, "to distinguish consciousness from unconscious mental computation," is impossible in absolute terms because it assumes that the distinction can be cleanly made. Consciousness is a spectrum, not a switch. We can subjectively distinguish degrees of consciousness.

The second part, to "identify its correlates in the brain," is also difficult because consciousness is a matter of degree. The forebrain seems to play a large part in awareness, but only a part.

We are unconscious of our retinas but not of the vision they give us, which begs the question of where along the neural path between eye and forebrain unconsciousness stops and consciousness begins. We are conscious of the words we say but unconscious of the motor processes producing the speech, which raises the question of where the boundary lies along the neural path between the forebrain and the larynx.

Consciousness can wander around the brain. While we are unconscious of our larynx when conversing, someone learning to speak a foreign language, or learning to sing, is not. For a ballet dancer learning a new work, consciousness moves from steps to choreography to nuances to performance; in performance, the lower levels become unconscious.

The third part, to "explain why it evolved," is much easier. It can be done in two stages: by explaining why the central nervous system evolved, and by explaining why consciousness evolved. Animals move around, plants do not. Animals have nervous systems, plants do not. A nervous system is needed to control movement. Relatively few animals (metazoa) have diffuse nervous systems; most-in

\section{We are born with an}

underdeveloped central nervous system,

and all our consciousness

is developed socially, or should be.

particular protostomes, which include insects, and deuterostomes, which include mammals-have a central nervous system. Indeed, the very early Urbilateria, from which both protoand deuterostomes descend, seems to have had such a system, one that sends sensory signals to the brain for it to process into motor signals (www. economist.com/science/PrinterFriendly. cfm?story_id=9033083). Likewise, the first digital computer I used, an IBM 650 , read data from cards, sent the data to the CPU for processing, then sent out the results to be punched into cards.

Simple animals have simple nervous systems, complex animals have complex ones. A nervous system controls its animal in its environment, sensing the surroundings, evaluating the sensations it receives, and commanding action. The action might be to feed, flee, freeze, fight, or-for sexual animals-fornicate. If the nervous system succeeds-a subjective term-the individual animal survives and propagates.

Are simple animals conscious? Certainly, they are aware of their surroundings and react to stimuli, so they are conscious to some degree. But because their brains are simple, they react in simple ways that might seem unconscious to us.
Complex animals evolved from simple ones, and they became complex because their bodies and their nervous systems became larger. Their senses, brains, and motor systems became more complex. Greater mobility meant richer sensation and required a more structured brain to process the sensations and to make more sensitive decisions about more complex potential behavior.

Evolution used parts of this structure to simplify and thus speed up decision making by storing patterns of sensation and behavior, which could be drawn upon unconsciously in commonly repeated situations. This freed up other parts to work with unconscious analyses of sensations and consciously select from available behaviors.

Analogous in the evolution of digital computers would be the cache's storing of more frequently used data, and the microprogram's storing of frequently used microprocesses. However, such analogies must not be carried too far. Nervous systems are not digital devices, nor do they separate signal processing from storing their content.

\section{THE HARD PROBLEM}

The Hard Problem is simply "explaining how subjective experience arises from neural computation," though if we give a strict meaning to the word compute, there is no neural computation. Our analog mental processes carry out mental not neural arithmetic, which combines conscious and unconscious thought, the proportions depending on the thinker's skill.

Subjective experience comes from social experience. Even in the womb, which is not a silent place, we learn about sound. We are born with an underdeveloped central nervous system, and all our consciousness is developed socially, or should be. The less social experience a child gets during infancy, the less its nervous system will develop, the poorer its consciousness will be, and the more difficult it will be for the adolescent to develop subjective capability.

A newborn child cannot see properly, and its visual experience causes its visual system to develop and refine. The 
stupendous subjectiveness of spoken language develops in an infant by social experience and, for an infant given the opportunity, it is as easy to learn several languages as it is to learn one. Speech, an important part of our subjective experience, is learned through conversation. Tragically, adverse family conditions and television's ready availability have meant that children starting school in developed countries sometimes are unable to converse, requiring remedial training before they can begin their formal education.

Education is, or should be, a cumulative social process that seeks to enrich subjectivity. Because conscious behavior is rich to the degree that the unconscious background is rich, effective education will convert as much conscious capability as possible into unconscious form. Thus, education should give priority to developing basic skills so that the student can consciously use more and more of them. The needed process is clear in special programs, such as those promoting excellence in music or athletics, but it is not often adopted in general education.

Because our consciousness is social, we have no useful analogy in digital computers. But there is another dimension of mentality to consider-a spectrum of styles of consciousness. At one end lies the consciousness that is entirely sensual, filled with sensation and reaction. A mainframe is like this when it is I/O-bound. At the other end lies the entirely contemplative consciousness, filled with evaluation and speculation. A mainframe is like this when it is compute-bound. An ideal consciousness will wander around the middle of this spectrum.

D igital technology has a role to play in education and has great potential to support the enrichment of consciousness. Unfortunately, it is much more often used in an unbalanced way. An example I read of when almost finished with this essay was so unbalanced as to be inhumane, and it brought tears to my eyes (www.theage. com.au/news/opinion/killing-soldiershumanity/2007/06/03/11808093363 64.html).

The computing profession has a social responsibility to ensure that digital technology enriches young people rather than encumbering them.

Neville Holmes is an honorary research associate at the University of Tasmania's School of Computing. Contact him at neville.holmes@utas.edu.au.Details of citations in this essay, and links to further material, are available at www. comp.utas.edu.au/users/nholmes/prfsn.

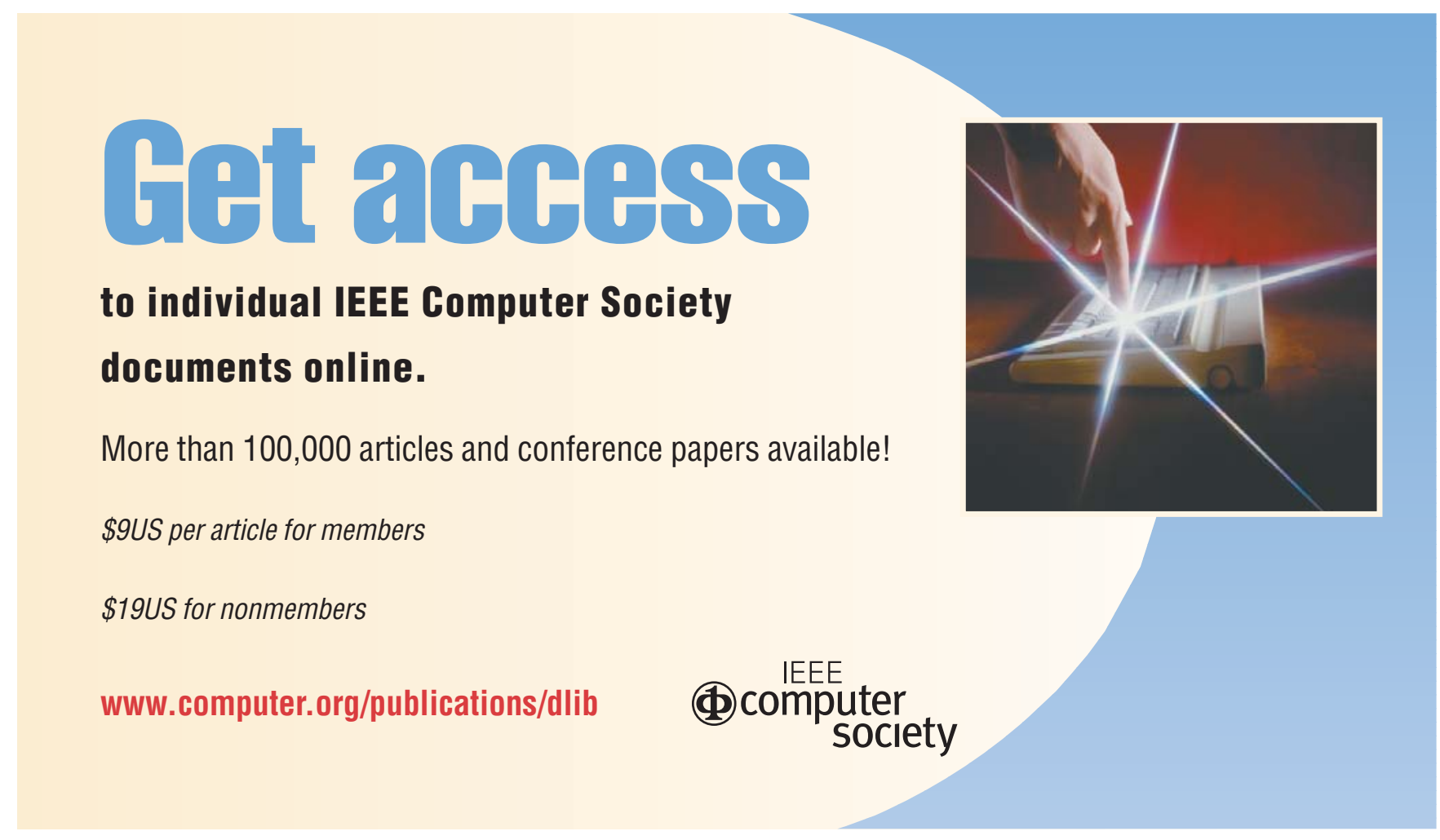

